\title{
How autism and Alzheimer's disease are TrAPPed
}

Debomoy K. Lahiri,1,2,3 Bryan Maloney,4,5 Ruizhi Wang,4 Deborah K. Sokol,6 Jack T. Rogers,7

\& Cara J. Westmark,8

${ }^{1}$ Department of Psychiatry and Stark Neuroscience Research Institute, Indiana University School of Medicine, Indianapolis, IN, USA. dlahiri@iupui.edu.

${ }^{2}$ Medical \& Molecular Genetics, Indiana University School of Medicine, Indianapolis, IN, USA. dlahiri@iupui.edu.

${ }^{3}$ Indiana Alzheimer's Disease Research Center, Indiana University School of Medicine, Indianapolis, IN, USA. dlahiri@iupui.edu.

${ }^{4}$ Department of Psychiatry and Stark Neuroscience Research Institute, Indiana University School of Medicine, Indianapolis, IN, USA.

${ }^{5}$ Indiana Alzheimer's Disease Research Center, Indiana University School of Medicine, Indianapolis, IN, USA.

${ }^{6}$ Department of Neurology, Indiana University School of Medicine, Indianapolis, IN, USA.

${ }^{7}$ Department of Psychiatry, Neurochemistry lab, MGH, Harvard Medical School, Charlestown, MA, USA.

${ }^{8}$ Department of Neurology, University of Wisconsin, Madison, WI, USA.

This Comment posits that two important psychiatric disorders, autism spectrum disorder (ASD) and Alzheimer's disease (AD), typical to opposite ends of the lifespan (early vs late), neuroanatomy (enlarged vs reduced amygdala), biochemistry (low vs high amyloid- $\beta$ peptide), and outcomes (neurotrophic vs neurodegeneration) [1], are molecularly in opposition specifically through metabolites of amyloid- $\beta$ precursor protein (APP). Herein, we attempt to address associated regulatory questions.

This is the author's manuscript of the work published in final edited form as:

Lahiri, D. K., Maloney, B., Wang, R., Sokol, D. K., Rogers, J. T., \& Westmark, C. J. (2021). How autism and Alzheimer's disease are TrAPPed. Molecular Psychiatry, 26(1), 26-29. https://doi.org/10.1038/s41380$\underline{020-00928-8}$ 
The divergent neuropsychiatric disorders ASD and AD have opposite molecular links that include divergent processing of APP. APP is commonly associated with AD through neurodegenerative processing products. The constitutive pathway of APP processing is neurotrophic and may contribute to early-life brain overgrowth typical of ASD, including traits such as macrocephaly (occurring in up to $20 \%$ of individuals with autism) [1] and increased brain volume vs non-autistic subjects that may persist up to 4 years of age [2]. Multiple protein factors regulate APP translation, including interleukin-1 (IL-1), iron regulatory protein-1 (IRP-1), and fragile-X mental retardation protein (FMRP). In addition, APP mRNA translation is also regulated by microRNA (miRNA) activity. Finally, net APP-derived activity is heavily influenced by mature protein cleavage, depending on whether the constitutive or amyloid pathway is followed. We suggest a potential novel avenue of scientific research, whereby APP translation is multiply TrAPPed (Translation of APP elevation and decline) and influences the etiology of ASD and AD. In short, the fates of ASD and AD are trapped in mis-regulation of APP mRNA transport, translation, and processing (Fig. 1A). As a result, it is the interaction of these pathways that likely influences the etiology and progression of neurodevelopmental and neurodegenerative disorders.

When considering contributing factors to AD, APP and its cleavage products are "usual suspects". This is through the amyloidogenic processing pathway, involving sequential proteolytic cleavages of APP by $\beta$ secretase ( $\beta$-site APP cleaving enzyme 1, BACE1) and $\gamma$-secretase complexes. The constitutive APP processing pathway, on the other hand, by sequential action of $\alpha$ - and $\gamma$-secretases, produces the secreted cleavage products SAPP $\alpha$ (and non-amyloidogenic p3 peptide), which function as neurotrophic factors that contribute to neurite growth and neuroproliferation, and synaptogenesis [3] and as an iron export protein [4]. This elevation of sAPP $\alpha$ associates with ASD [5]. Animal models also support a link. For example transgenic mice over-expressing sAPP $\alpha$, have elevated glial fibrillary acidic protein in brain and notable social deficits [6]. The amyloidogenic pathway generates the potentially toxic amyloid- $\beta$ (A $\beta$ ) peptide, which can aggregate in extraneuronal plaques in AD. However, A $\beta$ does not exist solely to cause disease and has normal neurological functions [7]. The constitutive pathway is important for 
neurodevelopmental disorders, such as ASD. Notably, p3 peptide (called "N-terminally truncated A $\beta$ ") is elevated in ASD [8], and A $\beta$ is reduced in ASD [9] but elevated in AD. The findings of sAPP $\alpha$ elevation [10] and $A \beta$ reduction [11] have been replicated by independent laboratories in human subjects with autism.

Importance of genetic mutations in a small number of disorders as "causes" has underplayed the importance of multiple steps downstream of the primary DNA sequence. Several mechanisms of such downstream regulation exist. For example, multiple specific miRNA species regulate APP protein expression post-transcriptionally, and these miRNAs are dysregulated in AD [12]. Such miRNA regulation of APP has gained considerable attention in recent years. Second, protein-based regulation of APP mRNA translation closely interacts with miRNA [13]. At least, two RNA-binding proteins (RBPs), specifically FMRP and heterogeneous ribonucleoprotein-C (hnRNPC), interact with APP mRNA in the coding region, in addition to IRP-1 and IL-1 binding to the APP 5'-untranslated region (5'-UTR) at the miR-346 binding site.

FMRP exercises two distinct modes of action in its regulation of mRNA transcripts. FMRP participates in nuclear export of mRNA by reading $N^{6}$-methyladenosine $\left(\mathrm{m}^{6} \mathrm{~A}\right)$ methylation [14]. FMRP's second method is to target guanine-rich (G-rich) coding sequences in mRNAs and inhibit protein synthesis. This second mechanism can inhibit APP protein synthesis. On the other hand, another $\mathrm{m}^{6} \mathrm{~A}$ reading protein, hnRNPC, upregulates protein synthesis of APP. Thus, hnRNPC and FMRP regulate APP protein synthesis in opposite directions [15]. Interestingly, even though both of these proteins function as $\mathrm{m}^{6} \mathrm{~A}$ reading proteins, their activity on $A P P$ is through competitive binding in the coding sequence, independently of $\mathrm{m}^{6} \mathrm{~A}$ modification [15].

Analysis of the effects of Fmrl knockout in model mice indicates that App mRNA transport was not affected [16]. That is, Fmrl knockout did not alter relative levels of nuclear vs. cytoplasmic App mRNA. On the other hand, hnRNPC activity on App mRNA transport is unknown, although the mechanism of FMRP downregulation versus hnRNPC upregulation is more likely to operate through competitive 
binding to G-rich regions [15]. Nonetheless, FMRP may contribute to transport of $\mathrm{m}^{6} \mathrm{~A}$-modified $\alpha$ secretase and $\gamma$-secretase messages, specifically A disintegrin and metalloproteinase-9 (Adam 9 ) and presenilin-1 (Psen1) mRNAs [16]. Differences in levels of resulting enzymes (along with other APP processing enzymes such as ADAM10/17 and BACE1) may alter the route of APP processing to either the anabolic or catabolic pathway [17].

APP processing products associate with both neurotrophy and neurodegeneration. Neurotrophic processing products associate with ASD conditions, characterized by neuronal overgrowth [9], while A $\beta$ associates with AD. Regarding the contribution of APP to autism, differential methylation of the mRNAs for APP processing enzymes could explain cases of ASD that do not accompany gross FMRP aberration. Thus, cross-talk among FMRP, hnRNPC, and secretase mRNAs could have implications for ASD and $\mathrm{AD}$ beyond G-quartet binding, as well as more uncommon but related disorders, such as fragile $\mathrm{X}$ syndrome (FXS), Down syndrome [18], and the "orphan disease” DUP-APP [19].

If the balance of total secreted APP (sAPP) and A $\beta$ is disrupted by FMRP and hnRNPC then we would predict iron balance would also be disrupted. Upsetting iron balance may affect neuron density and interconnection and may contribute to autism. sAPP $\alpha$ induces an increase in glutamatergic and a decrease in GABA-ergic synapses creating an excitatory/inhibitory imbalance observed in autism. sAPP $\alpha$ in the brain may further affect the GABAergic regulations by suppressing presynaptic vesicle release through direct binding of sAPP extension domain to the GABA type B [20]. This may be another APP-related mechanism of GABAergic dysregulation in autism. Notably, while elevated levels of sAPP $\alpha$ and diminished A $\beta$ have been found in autism patient brains, elevation of overall APP was not found to be typical of autism, although it was found in FXS [9].

Future studies would warrant measuring the effects of other RBPs in $\mathrm{m}^{6} \mathrm{~A}$-mediated transport studies, aside from confirming Fmrl knockout [16]. While $\mathrm{m}^{6} \mathrm{~A}$ reading by FMRP certainly influences how much of a given mRNA enters the cytoplasm, there is more to protein synthesis regulation than mRNA levels and location. APP translation is also regulated via interactions between RBPs, iron response elements and 
microRNA-346 in the 5'-UTR of APP mRNA [13], by cytokines such as interleukin-1 [13]. Thus, translational regulation of APP and secretases via mRNA binding proteins, microRNA and/or methylation of messenger RNA could explain the etiology of some cases of brain disorders. In short, these pathways could potentially emerge as a novel avenue for translational research whereby APP translation and processing are multiply TrAPPed among disparate pathways (Fig. 1). Further investigation of this novel link in human subjects with APP-related disorders could help in understanding AD, ASD, and other psychiatric diseases. In addition, seizures and EEG abnormalities in fragile X and ASD might accompany abnormal levels of APP metabolites. Genetic suppression of transgenic APP in mice suggests that APP processing may be a potential contributor to seizure activity [21]. While beyond the editorial limits of a short commentary, health issues as disparate as inflammation and iron dyshomeostasis have also been seen in association with ASD and AD. How they might differ between the two conditions warrants further investigation. 


\section{References}

1. McCaffery P, Deutsch CK. Macrocephaly and the control of brain growth in autistic disorders. Prog Neurobiol. 2005;77: 38-56.

2. Mosconi MW, Cody-Hazlett H, Poe MD, Gerig G, Gimpel-Smith R, Piven J. Longitudinal study of amygdala volume and joint attention in 2- to 4-year-old children with autism. Arch Gen Psychiatry. 2009;66:509-16.

3. Mattson MP. Secreted forms of beta-amyloid precursor protein modulate dendrite outgrowth and calcium responses to glutamate in cultured embryonic hippocampal neurons. J Neurobiol. 1994;25:43950.

4. Tsatsanis A, Wong BX, Gunn AP, Ayton S, Bush AI, Devos D, et al. Amyloidogenic processing of Alzheimer's disease beta-amyloid precursor protein induces cellular iron retention. Mol Psychiatry. 2020;25:1958-66.

5. Ray B, Long JM, Sokol DK, Lahiri DK. Increased secreted amyloid precursor protein-alpha (sAPPalpha) in severe autism: proposal of a specific, anabolic pathway and putative biomarker. PLoS One. 2011;6:e20405.

6. Bailey AR, Hou H, Song M, Obregon DF, Portis S, Barger S, et al. GFAP expression and social deficits in transgenic mice overexpressing human sAPPalpha. Glia. 2013;61:1556-69.

7. Lahiri DK, Maloney B. Beyond the signaling effect role of amyloid- $\beta 42$ on the processing of APP, and its clinical implications. Exp Neurology. 2010;51-4.

8. Frackowiak J, Mazur-Kolecka B, Mehta P, Wegiel J. Enhanced accumulation of N-terminally truncated $A \beta$ with and without pyroglutamate- 11 modification in parvalbumin-expressing GABAer-gic neurons in idiopathic and dup15q11.2-q13 autism. Acta Neuro-pathol Commun. 2020;8:58. 
9. Ray B, Sokol DK, Maloney B, Lahiri DK. Finding novel distinctions between the sAPP $\alpha-$ mediated anabolic biochemical pathways in Autism Spectrum Disorder and Fragile X Syndrome plasma and brain tissue. Sci Rep. 2016;6:26052.

10. Bailey AR, Giunta BN, Obregon D, Nikolic WV, Tian J, Sanberg CD, et al. Peripheral biomarkers in autism: secreted amyloid precursor protein-alpha as a probable key player in early diagnosis. Int J Clin Exp Med. 2008;1:338-44.

11. Carter CJ. Autism genes and the leukocyte transcriptome in autistic toddlers relate to pathogen interactomes, infection and the immune system. A role for excess neurotrophic sAPP $\alpha$ and reduced antimicrobial A $\beta$. Neurochem Int. 2019; 126:36-58.

12. Long JM, Ray B, Lahiri DK. MicroRNA-153 physiologically inhibits expression of amyloid- $\beta$ precursor protein in cultured human fetal brain cells and is dysregulated in a subset of Alzheimer disease patients. J Biol Chem. 2012;287:31298-310.

13. Long JM, Maloney B, Rogers JT, Lahiri DK. Novel upregulation of amyloid-beta precursor protein (APP) by microRNA-346 via targeting of APP mRNA 5'-untranslated region: implications in Alzheimer's disease. Mol Psychiatry. 2019;24:345-63.

14. Hsu PJ, Shi H, Zhu AC, Lu Z, Miller N, Edens BM, et al. The RNA-binding protein FMRP facilitates the nuclear export of N6-methyladenosine-containing mRNAs. J Biol Chem. 2019; 294:19889-95. 
Fig. 1: Autism and Alzheimer's disease-APP function TrAPPed in place.

A

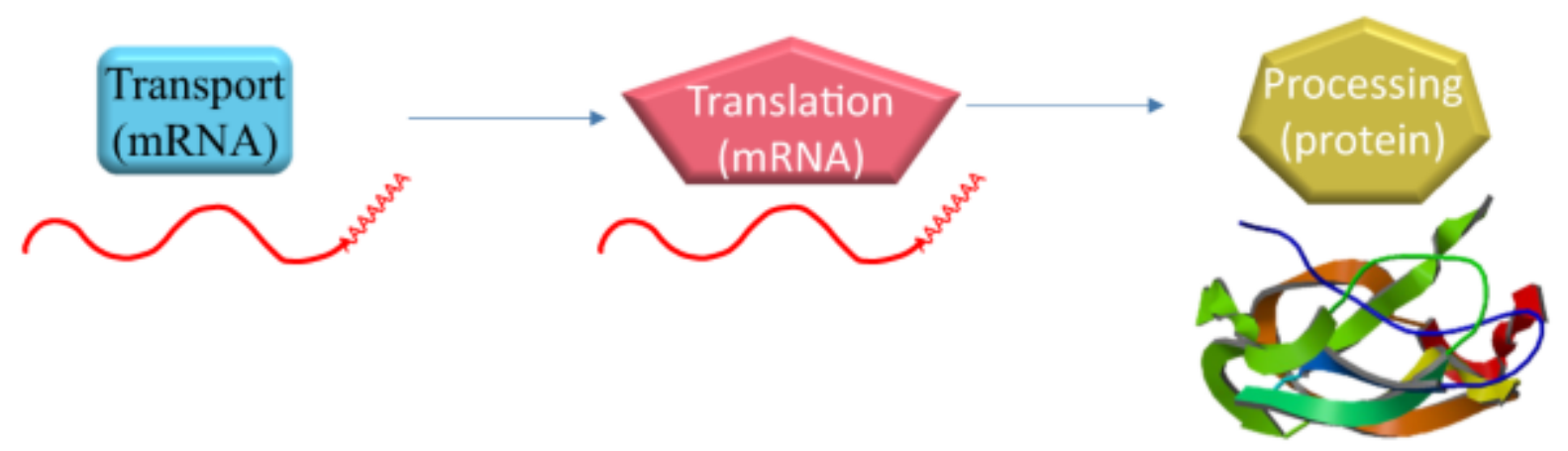

B

APP mRNA

G-quartet

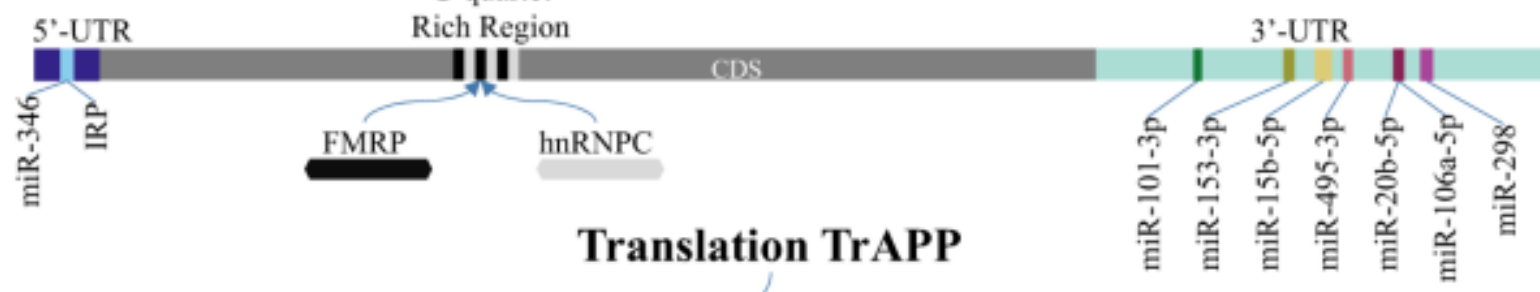

mRNA Transport TrAPP
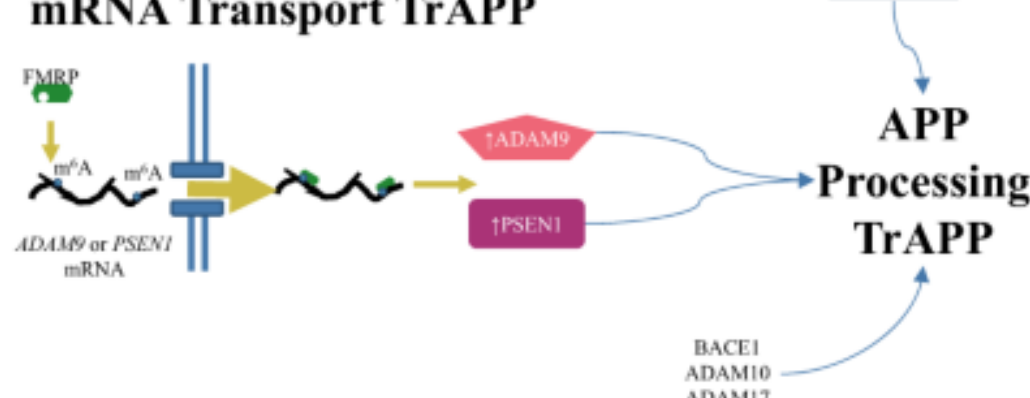

APP

(protein)

TrAPP

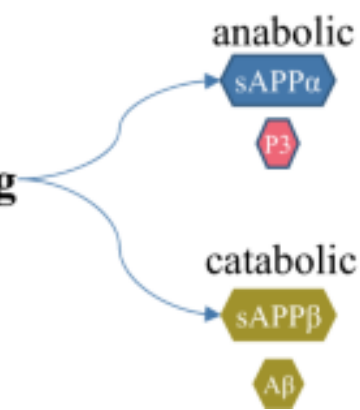

Autism

(ASD)

Alzheimer's

(AD)

A Schematic diagram of the "3-Way TrAPP”. Levels of final processed APP products are influenced by the product of translation regulation of APP mRNA by mRNA binding proteins and microRNA, and by levels of APP processing enzymes, which are in part regulated by mRNA transport across the nuclear membrane due to differential $\mathrm{m}^{6} \mathrm{~A}$ methylation. B Production of APP and its cleavage into functional subunits is held in homeostasis by a "TrAPP" of translational control of APP mRNA and transport regulation of processing enzymes. Translation is controlled in part by protein factor binding to the APP 
mRNA, which includes both competitive repression/derepression from FMRP and hnRNPC and repression by binding of IRP1. IRP1 repression interacts with miR346 stimulation. Several miRNA species likewise bind the APP mRNA to repress or stimulate translation. Multiple repressive bindings occur in the APP 3'-UTR, while miR346 stimulates APP translation in competition against the repressive activity of IRP. Finally, transport of selected APP processing enzymes, such as ADAM9 and PSEN1, to the cytoplasm for translation are under the control of FMRP binding to $\mathrm{m}^{6} \mathrm{~A}$ modification of the respective mRNAs. Differences in levels of resulting enzymes may modify (along with other APP processing enzymes such as ADAM10/17 and BACE1) the emphasis of APP processing to anabolic or catabolic pathways. 